\title{
BINARY STAR FORMATION AND MASS OUTFLOWS: MHD NESTED GRID SIMULATION
}

\author{
M. N. Machida, ${ }^{1,2}$ K. Tomisaka, ${ }^{2}$ and T. Matsumoto ${ }^{3}$
}

\begin{abstract}
RESUMEN
Estudiamos el proceso de formación estelar a partir de una nube molecular magnetizada y en rotación. Partimos de una nube cilíndrica isotérmica en equilibrio hidrostático, cuyo eje de rotación coincide con las líneas del campo magnético global, y es paralelo al eje del cilindro. Añadimos perturbaciones de densidad axisimétricas y no axisimétricas al estado inicial, y estudiamos la evolución subsecuente. La evolución se caracteriza por tres parámetros: la amplitud de las perturbaciones no axisimétricas, la velocidad de rotación y la intensidad del campo magnético. Encontramos que la desviación de la axisimetría apenas cambia en la fase inicial, pero que empieza a crecer después de que el gas se contrae y forma un disco delgado. Hay dos tipos de fragmentación: a partir de un anillo y de una barra. Los núcleos adiabáticos delgados se fragmentan si su espesor es menor que $1 / 4$ del radio. Para que los fragmentos sobrevivan, deben formarse en un núcleo muy elongado, o en un disco plano. En los modelos con fragmentación se encuentran flujos emanando tanto de los fragmentos como de la barra en rotación o del disco.
\end{abstract}

\begin{abstract}
We study the binary star formation process from a rotating magnetized molecular cloud. We assume an isothermal cylindrical cloud in hydrostatic balance whose rotation axis and the direction of global magnetic field lines are both identical, and parallel to the cylinder axis. We added axisymmetric and non-axisymmetric density perturbations to the initial state and followed the subsequent evolutions. The evolution is characterized by three parameters: the amplitude of the non-axisymmetric perturbations, the rotation speed, and the magnetic field strength. As a result, it is found that non-axisymmetry hardly evolves in the early phase, but begins to grow after the gas contracts and forms a thin disk. There are two types of fragmentation: fragmentation from a ring and that from a bar. Thin adiabatic cores fragments if a thickness is smaller than $1 / 4$ of the radius. For the fragments to survive, they should be formed in a heavily elongated barred core or a flat round disk. In the models showing fragmentation, outflows from respective fragments are found as well as those driven by the rotating bar or the disk.
\end{abstract}

\section{Key Words: BINARIES: GENERAL - ISM: JET AND OUTFLOWS - ISM: MAGNETIC FIELDS - STARS: FORMATION}

\section{INTRODUCTION}

It is important to understand how a binary star system forms from a molecular gas cloud because most main-sequence stars are in binary or multiple systems (e.g. Abt 1983; Duquennoy \& Mayor 1991). Furthermore recent observations indicate that companions may be even more common for pre-main sequence stars (Richichi et al. 1994). Numerical simulations show that it is difficult for single stars to capture another one and to form a binary system in the main sequence phase (Kroupa \& Burkert 2001). Accordingly, most stars seem to be born as binaries rather than single stars in a gas cloud, and molecular bipolar outflows are often observed

\footnotetext{
${ }^{1}$ Hokkaido University.

${ }^{2}$ National Astronomical Observatory, Japan.

${ }^{3}$ Hosei University.
}

in star forming regions. It has been shown using two-dimensional magnetohydrodynamical simulations that outflow plays an important role in the star formation process because the excess angular momentum, which is conserved from the host cloud, is removed by these outflows from the formed core (Tomisaka 2000; 2002). These outflows are caused by the twisted magnetic field lines made by the rotation near the adiabatic core. In order to investigate the cloud fragmentation and later binary star formation, three-dimensional calculation is required.

In this paper, we discuss fragmentation in the course of star formation which leads to binary star formation. In order to explore the fragmentation of the magnetized cloud, full three-dimensional magnetohydrodynamical (MHD) simulations are necessary. Here, we employ the nested grid MHD code, 
which always maintains sufficient spatial resolution in the central region. The nested grid method is a powerful means of studying the star formation process, because this requires a large dynamical range in spatial dimensions. In this study, we calculate the non-axisymmetric dynamical contraction of the cloud from $4 \times 10^{2} \mathrm{~cm}^{-3}$ to $1 \times 10^{17} \mathrm{~cm}^{-3}$ in density, and investigate how fragmentation proceeds and outflow occurs in the course of binary star formation.

\section{MODEL AND NUMERICAL METHOD}

To study the non-axisymmetric evolution (binary star formation and outflow), we consider a cylindrical isothermal cloud in hydrostatic balance as the initial condition. The cloud is assumed to rotate parallel with a rotation axis which coincides with the cylindrical axis and the magnetic field lines are assumed to run parallel to the rotation axis. We added perturbation of the length, $\lambda$, (Matsumoto, Nakamura \& Hanawa 1994) to the infinitely long cylinder.

Using the cylindrical coordinates $(r, \phi, z)$, the density, azimuthal velocity, and magnetic flux density distributions in the radial direction are taken to be as follows (Stodółkiewicz 1963):

$$
\begin{aligned}
\rho_{0}(r) & =\rho_{c}\left[1+\left(r^{2} / 8 H^{2}\right)\right]^{-2}, \\
v_{\phi 0}(r) & =r \Omega_{c}\left[1+\left(r^{2} / 8 H^{2}\right)\right]^{-1 / 2}, \\
B_{z 0}(r) & =B_{c}\left[1+\left(r^{2} / 8 H^{2}\right)\right]^{-1},
\end{aligned}
$$

where $\rho_{c}, \Omega_{c}$, and $B_{c}$ represent the density, angular rotation speed, and magnetic flux density at the center of the cylindrical cloud, respectively, and $H$ is the scale-height as $H^{2}=\left(c_{s}^{2}+B_{c}^{2} / 8 \pi \rho_{c}\right) /\left(4 \pi G \rho_{c}-2 \Omega_{c}^{2}\right)$, where $c_{s}$ denotes the isothermal sound speed. Molecular gas obeys the isothermal equation of state below $\rho_{\text {cri }} \approx 10^{10} \mathrm{~cm}^{-3}$ but it becomes adiabatic if $\rho \gtrsim \rho_{\text {cri }}$ (Tohline 1982). To mimic this, we adopt a two-component equation of state as $P=c_{s}^{2} \rho+$ $c_{s}^{2} \rho_{\text {cri }}\left(\rho / \rho_{\text {cri }}\right)^{7 / 5}$.

The initial central density and the critical density are chosen as $\rho_{c 0}=4 \times 10^{2} \mathrm{~cm}^{-3}$ and $\rho_{\text {cri }}=10^{10} \mathrm{~cm}^{-3}$. We added axisymmetric and nonaxisymmetric perturbations to the above magnetohydrostatic equilibrium as

$$
\begin{gathered}
\rho(r, z, \phi)=\rho_{0}(r)\left[1+\delta \rho_{z}(z)\right]\left[1+\delta \rho_{\phi}(r, \phi)\right] \\
B_{z}(r, z, \phi)=B_{z 0}(r, \phi)\left[1+\delta B_{\phi}(r, \phi)\right]
\end{gathered}
$$

with

$$
\begin{aligned}
\delta \rho_{z}(z) & =A_{z} \cos (2 \pi z / L), \\
\delta \rho_{\phi}(r, \phi) & =\left\{\begin{array}{l}
A_{\phi}(r / H)^{2} \cos (m \phi), \text { for } r_{\leq} H, \\
A_{\phi} \cos (m \phi), \text { for } r>H .
\end{array}\right.
\end{aligned}
$$

In this paper, we restrict ourselves to the relative amplitude of the axisymmetric perturbation $A_{z}=0.1$. As the non-axisymmetric perturbation, only the $m=2$ mode is included. Models are parameterized with three non-dimensional parameters: the amplitude of the non-axisymmetric perturbation as $A_{\phi}$, the magnetic-to-thermal pressure ratio as $\alpha\left(\equiv B_{c}^{2} / 4 \pi \rho_{c} c_{s}^{2}\right)$ and the angular speed normalized by the free-fall timescale as $\omega \equiv \Omega_{c} /\left(4 \pi G \rho_{c}\right)^{1 / 2}$. We calculated 51 different models with $A_{\phi}=$ $(0,0.01,0.1,0.2), \alpha=(0,0.01,0.1,1,5)$ and $\omega=(0,0.1,0.5,0.7)$.

In order to solve the central region with higher spatial resolutions, we adopt an MHD nested grid method based on the Cartesian coordinate (for details, see Machida 2003). In this method, a number of grids with different spacings are prepared, in which finer grids cover the central high-density portion and the coarser ones cover the cloud as a whole. Each grid has successively different cell widths by a factor two. We use $128 \times 128 \times 32$ cubic cells in the $x-, y$ - and $z$-directions. We added a new finer grid to maintain the Jeans condition, $\lambda_{J} / 4>h$, with an ample margin (Truelove et al. 1997), where $\lambda_{J}$ and $h$ are the Jeans length and the cell width, respectively. Only when a high density fragment escapes from the region covered by the finest grid, the Jeans condition is violated in our simulations.

\section{RESULTS}

The cylindrical gas cloud collapses to become spherical in shape in the early collapse phase. If the initial gas cloud has neither magnetic field nor rotation speed, the cloud continues to collapse spherically and it forms into a small massive core in the center of the cloud (Larson 1969) even in a cylindrical cloud (Tomisaka 1995). In the case of a gas cloud with magnetic fields or rotation, it collapses to form a pseudo-disk due to the magnetic pressure or centrifugal force (Tomisaka 1995; Nakamura \& Hanawa 1997; Matsumoto, Hanawa \& Nakamura 1997). If only the axisymmetric perturbation exists, a round disk is formed, but it does not fragment as long as the axisymmetry holds. When the non-axisymmetric perturbation is added, the cloud evolves to form a non-axisymmetric shape, and fragments later for some range of the parameters. In any case, the central density $\left(\rho_{c}\right)$ increases with time and the gas becomes adiabatic when $\rho_{c}>10^{10} \mathrm{~cm}^{-3}$. For convenience, we divide the evolution into two phases: isothermal phase $\left(\rho_{c}<10^{10} \mathrm{~cm}^{-3}\right)$ and adiabatic phase $\left(\rho_{c}>10^{10} \mathrm{~cm}^{-3}\right)$. The outflow is driven by the twisted magnetic fields (Tomisaka 1998; 2002) 


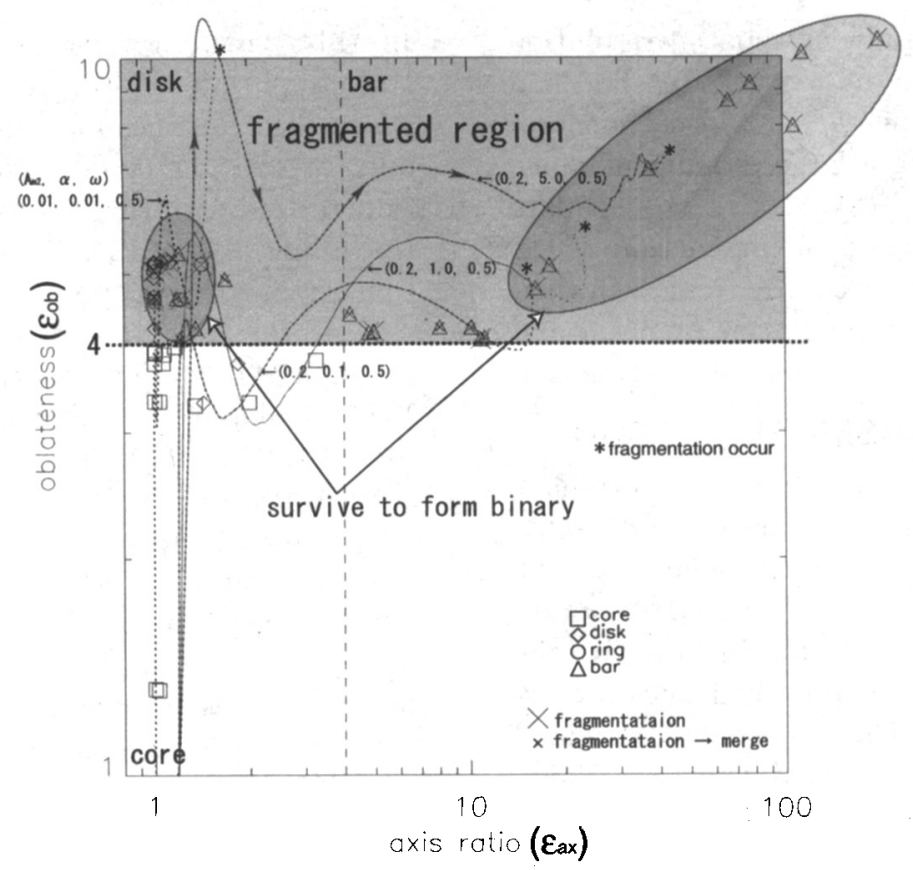

Fig. 1. The axis ratio and oblateness are plotted when the gas at the cloud center becomes adiabatic $\rho_{c}=\rho_{\text {cri }}$. The abscissa indicates the axis-ratio and the ordinate is the oblateness. Both are calculated for the gas with $\rho>(1 / 10) \rho_{\max }$. Crosses represent cores which experience fragmentation. Fragmentation occurs above the horizontal dashed line $(\varepsilon>4)$. The domains in large circles mean that fragmented cores survive to form binary or multiple stars. The solid lines represent the evolutionary tracks for some typical parameters. Dotted lines represent the evolution after the adiabatic core is formed. Asterisks show the points of fragmentation. The symbols denote the shape of the adiabatic core when the fragmentation occurs or the calculation ends.

after $\sim 10^{3}$ yr have passed from the core formation epoch $\rho_{c}=\rho_{\text {cri }}$. Modes of fragmentation and shapes of the outflow depend on the growth of nonaxisymmetry. This shows that the cloud evolution can be classified by this growth. In order to characterize the shape of the central region, we define oblateness $\left(\varepsilon_{\mathrm{ob}}\right)$ and axis ratio $\left(\varepsilon_{\mathrm{ar}}\right)$ as follows:

$$
\begin{aligned}
\varepsilon_{\mathrm{ob}} & \equiv \sqrt{h_{l} \cdot h_{s}} / h_{z}, \\
\varepsilon_{\mathrm{ar}} & \equiv h_{l} / h_{s},
\end{aligned}
$$

where $h_{l}, h_{s}$ and $h_{z}$ mean the length of the long-, short-, and $z$-axis for the gas with $\rho>(1 / 10) \rho_{\max }$, respectively.

In Figure 1, the oblateness and the axis ratio of the central region are plotted for all models at the beginning of the adiabatic phase $\left(\rho_{\mathrm{c}}=\rho_{\mathrm{cri}}=\right.$ $10^{10} \mathrm{~cm}^{-3}$ ). Curves in this figure illustrate the evolutionary tracks for four different models $\left(A_{\phi}, \alpha, \omega\right)=$ $(0.01,0.01,0.5),(0.2,0.1,0.5),(0.2,1,0.5)$, and $(0.2,5,0.5)$. The clouds evolve from the lowerleft corner, which means the early shape is almost spherical, to the upper-right region via the upper-left region. First, the clouds evolve vertically upward.
This indicates that the oblateness increases. Then, the curves change their directions to the right for three models with $A_{\phi}=0.2$. Evolutionary tracks of the high-density region in Figure 1 show very clearly that the non-axisymmetric perturbation grows only after the disk is formed. That is, the axis ratio increases after the oblateness grows considerably large $\left(\varepsilon_{\mathrm{ob}} \geq 4\right)$. Because the gas is partially supported in the radial direction by magnetic pressure and centrifugal force, the radial contraction is more delayed than that of the $z$-direction. As a result, the disk is formed $\left(\varepsilon_{\mathrm{ob}}>4\right)$ earlier for models with larger $\alpha$ and $\omega$. In such models, the non-axisymmetry grows sufficiently and forms a large bar in the isothermal phase. However, the non-axisymmetry hardly evolves in the model with small $\alpha$ and $\omega$, because it takes a long time for disk formation.

Adiabatic cores are classified into three types: core $\left(\varepsilon_{\mathrm{ob}}<4\right.$ and $\left.\varepsilon_{\mathrm{ar}}<4\right)$, disk $\left(\varepsilon_{\mathrm{ob}}>4\right.$ and $\left.\varepsilon_{\mathrm{ar}}<4\right)$, and bar $\left(\varepsilon_{\mathrm{ob}}>4\right.$ ans $\left.\varepsilon_{\mathrm{ar}}>4\right)$. Some disks evolve into the rings, in which the central density is lower than that outside, even if they have the same oblateness and the axis ratio. Models with a 
cross $(x)$ experience fragmentation. In the models with small crosses, the fragments finally merge with each other before the calculation ends. On the other hand, the large cross means that the fragments survive without merger at the end of the calculation. Since the models with crosses are distributed only in the region $\varepsilon_{\mathrm{ob}}>4$, it is concluded that the necessary condition for fragmentation is $\varepsilon_{\mathrm{ob}}>4$, which means that a thin disk or bar is necessary for fragmentation. It is concluded that for the adiabatic core to fragment, the central gas needs to form a sufficiently thin disk or bar in the isothermal phase. The large crosses are distributed in two regions enclosed by circles. The fragmented cores in the circles seem to evolve into binary stars because the length of the semi-major axis expands or oscillates. Models in which the fragments merge with each other are distributed outside the domain. Outside the domain, a few fragments survive at the end of the calculation but the separation between the two cores shrinks with time. These will not grow into binary stars.

\section{CONCLUSION}

In conclusion, if the following criteria are fulfilled at the end of the isothermal phase, fragmentation occurs and fragments survive to form binary or multiple stars.

1. $\varepsilon_{\mathrm{ob}}>4$ : fragmentation condition.

2. $0<\varepsilon_{\text {ar }}<2$, or $\varepsilon_{\text {ar }}>10$ : survival condition against merger.

The symbol in this figure indicates the shape of the adiabatic core when the fragmentation occurs or the calculation ends. Figure 1 confirms the idea that the fragmentation occurs from a ring or bar and not from a core.
We showed the domain which may evolve into binary stars in Figure 1. Outside the binary-forming domain, the core formation model results in a compact massive core at the center of the cloud. The barfragmentation model in which the mutual merger of the fragments occurs also leads to a disk-like core at the center of the cloud. This configuration seems to lead to single star formation.

We have greatly benefited from discussion with Prof. T. Hanawa and Dr. H. Koyama. Numerical calculations were carried out by Fujitsu VPP5000 at the Astronomical Data Analysis Center, the National Astronomical Observatory of Japan.

\section{REFERENCES}

Abt, H. A. 1983, ARA \& A, 21, 343

Duquennoy, A., \& Mayor, M. 1991, A\&A, 248, 485

Larson, R. B. 1969, MNRAS, 145, 271.

Machida, N. M. 2003, PhD thesis, University of Hokkaido Matsumoto, T., Nakamura, F., \& Hanawa, T. 1994, PASJ, 46, 243

Matsumoto, T., Hanawa, T., \& Nakamura, F. 1997, ApJ, 478,569

Nakamura, F., \& Hanawa, T. 1997, ApJ, 480, 701

Kroupa, P., \& Burkert, A. 2001, ApJ, 555, 945

Richichi, A., Leinert, Ch., Jameson, R., \& Zinnecker, H. 1994. A\&A, 287, 145.

Stodółkiewicz, J. S. 1963, Acta Astron., 13, 30

Tohline, J. E. 1982, Fund. Cosm. Phys., 8, 1

Tomisaka, K. 1995, ApJ, 438, 226

Tomisaka, K. 1998, ApJ, 502, L163

Tomisaka, K. 2000, ApJ, 528, L41

Tomisaka, K. 2002, ApJ, 575, 306

Truelove, J, K., Klein, R. I., McKee, C. F., Holliman, J. H., Howell, L. H., \& Greenough, J. A. 1997, ApJ, 489, L179

Masahiro N. Machida and Kohji Tomisaka: Division of Theoretical Astrophysics, National Astronomical Observatory Japan, 2-21-1 Osawa, Mitaka, Tokyo 181-8588, Japan.

Tomoaki Matsumoto: Department of Humanity and Environment, Hosei University, Fujimi, Chiyoda-ku, Tokyo 102-8160, Japan. 


\section{DISCUSSION}

Scarfe - You chose $\gamma=7 / 5$, for a diatomic gas. Why?

Machida - I assume only $H_{2}$ gas, because we assumed a low temperature gas.

Guinan - Your modeling and simulations using MHD codes are excellent. In addition to the formation of stars and multiple stars, it also can be applied to galaxy and multiple galaxy systems. Have you thought about applying your work to galaxies?

Machida - No, not yet.

Rodriguez - Is the outflow you produce something transient or does it remain for some time?

Machida - In my calculation, the outflow is strongly driven. We stop calculating after the outflow becomes weak.

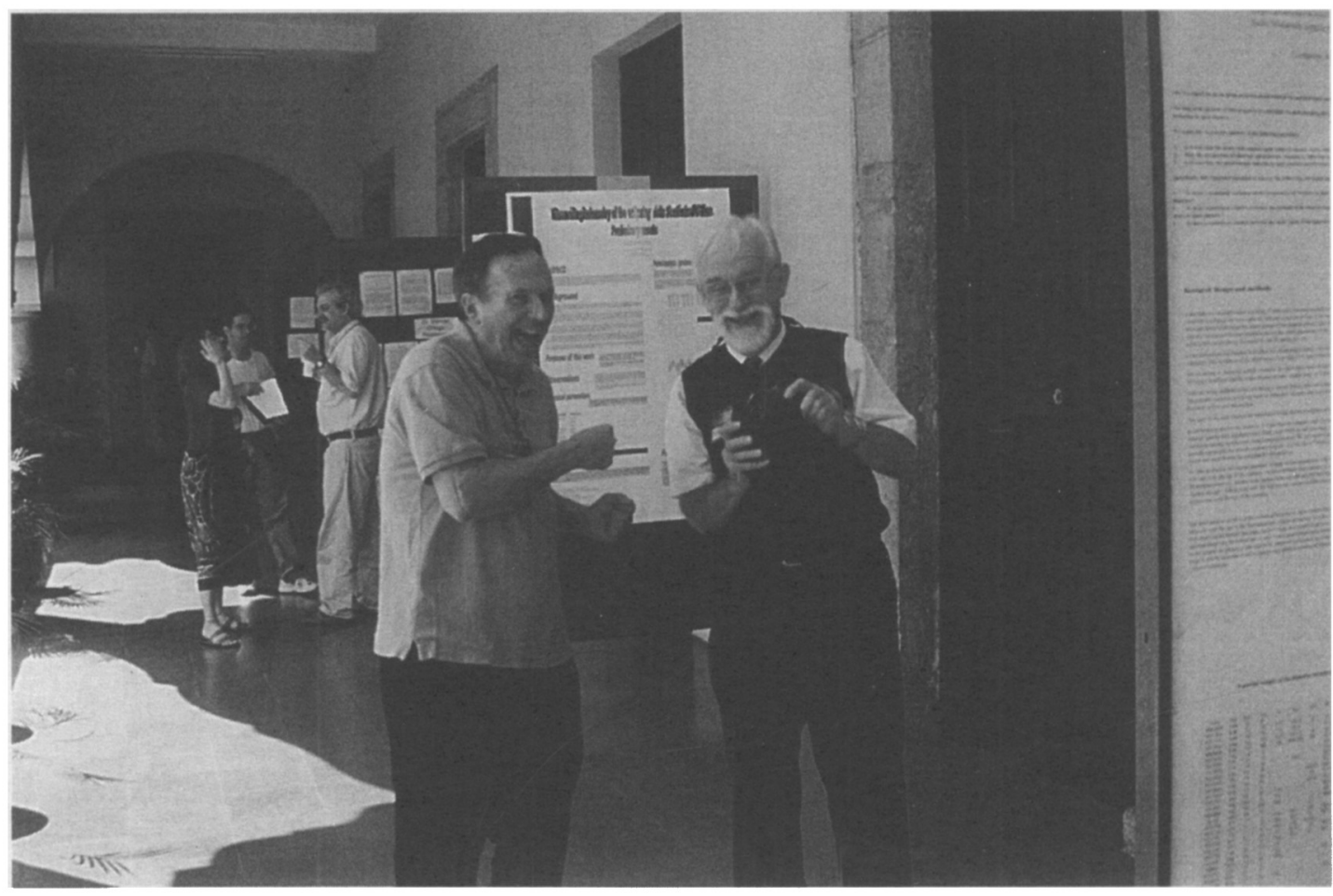

Ed Guinan and Colin Scarfe. 